\title{
Prediction consistency and clinical presentations of breast cancer molecular subtypes for Han Chinese population
}

\author{
Chi-Cheng Huang 1,2,3,4, Shih-Hsin Tu, 4, Heng-Hui Lien ${ }^{3,5}$, Jaan-Yeh Jeng ${ }^{2,3,4}$, Jung-Sen Liu, ${ }^{3,5}$, Ching-Shui Huang ${ }^{4,5}$, \\ Yih-Yiing Wu ${ }^{6}$, Chih-Yi Liu ${ }^{6}$, Liang-Chuan Lai', Eric Y Chuang ${ }^{1 *}$
}

From Organisation for Oncology and Translational Research (OOTR) 7th Annual Conference Hong Kong. 13-14 May 2011

\begin{abstract}
Background: Breast cancer is a heterogeneous disease in terms of transcriptional aberrations; moreover, microarray gene expression profiles had defined 5 molecular subtypes based on certain intrinsic genes. This study aimed to evaluate the prediction consistency of breast cancer molecular subtypes from 3 distinct intrinsic gene sets (Sørlie 500, Hu 306 and PAM50) as well as clinical presentations of each molecualr subtype in Han Chinese population.

Methods: In all, 169 breast cancer samples (44 from Taiwan and 125 from China) of Han Chinese population were gathered, and the gene expression features corresponding to 3 distinct intrinsic gene sets (Sørlie 500, Hu 306 and PAM50) were retrieved for molecular subtype prediction.

Results: For Sørlie 500 and Hu 306 intrinsic gene set, mean-centring of genes and distance-weighted discrimination (DWD) remarkably reduced the number of unclassified cases. Regarding pairwise agreement, the highest predictive consistency was found between Hu 306 and PAM50. In all, 150 and 126 samples were assigned into identical subtypes by both Hu 306 and PAM50 genes, under mean-centring and DWD. Luminal B tended to show a higher nuclear grade and have more HER2 over-expression status than luminal A did. No basal-like breast tumours were ER positive, and most HER2-enriched breast tumours showed HER2 over-expression, whereas, only two-thirds of ER negativity/HER2 over-expression tumros were predicted as HER2-enriched molecular subtype. For 44 Taiwanese breast cancers with survival data, a better prognosis of luminal A than luminal B subtype in ERpostive breast cancers and a better prognosis of basal-like than HER2-enriched subtype in ER-negative breast cancers was observed.

Conclusions: We suggest that the intrinsic signature Hu 306 or PAM50 be used for breast cancers in the Han Chinese population during molecular subtyping. For the prognostic value and decision making based on intrinsic subtypes, further prospective study with longer survival data is needed.
\end{abstract}

\section{Background}

In the past decade, microarray experiments have redefined breast cancers as heterogeneous diseases in terms of transcriptional aberrations, and a number of taxonomic classifications based on gene-expression profiles that have been reported have shown some prognostic significance. One

\footnotetext{
* Correspondence: chuangey@ntu.edu.tw

'Graduate Institute of Biomedical Electronics and Bioinformatics, National

Taiwan University, Taipei City, Taiwan

Full list of author information is available at the end of the article
}

such molecular taxonomy is the 'intrinsic subtype' proposed by the Stanford group. Perou identified 476 intrinsic genes from 65 patients with breast cancers and normal individuals; four subclasses: basal-like, Erb-B2+, normal breast-like, and luminal epithelial/ER+ were revealed by class discovery through clustering analysis $[1,2]$. The luminal subtype was further divided into luminal $A$ and $B$, and distant metastases were strongly associated with the expression patterns of intrinsic genes [3]. Independent studies supporting the existence of breast cancer intrinsic
C Biomed Central 
subtypes followed $[4,5]$. By definition, intrinsic genes were those genes that show the highest variation across different subjects and show the least variation within each individual (i.e. pre-/post-chemotherapy changes) [3]. The latest version of intrinsic signatures, prediction analysis of microarray 50 gene set (PAM50), was supposed to provide prognostic and predictive values independent of traditional prognostic factors such as hormone receptor, human epidermal growth factor receptor 2 (HER2) over-expression, or proliferation markers [6].

Although much attention has been drawn and intense arguments have been made, serious concerns about the true existence and reproducibility of intrinsic signatures remain. For instance, Lusa debated the comparability of study populations and concluded that assigning of new samples, which were not a part of the original dataset from which the intrinsic genes were derived to molecular subtypes was elusive [7]. Recently, Weigelt compared the agreement in subtype assignment across 3 different intrinsic genes-based single sample predictor (SSP) and found only fair to substantial pairwise agreement [8]. Indeed, the degree of overlap between distinct intrinsic gene lists was surprisingly low, although most of these molecular signatures were claimed to have some prognostic values [9]. The reproducibility and robustness of molecular subtypes from intrinsic genes by hierarchical clustering was also challenged [7,10].

In the current study, we evaluated the application of molecular subtypes across ethnic groups. Predictive consistency across different intrinsic gene sets as well as the impact of systemic microarray bias adjustment was assessed for Taiwanese and Chinese breast cancer patients, both of Han Chinese origin. Clinical and pathological features of each molecular subtype were compared accordingly.

\section{Methods}

\section{Study population and microarray experiments}

The study material included 169 breast cancers of Han Chinese population; 44 were from Taiwan, and 125, from Mainland China. Regarding the Taiwanese samples, sporadic breast cancer samples were collected consecutively during surgery, snapped frozen in liquid nitrogen, and then stored and transported at $-80^{\circ} \mathrm{C}$ from January 2007 to January 2008. The frozen samples were dissected into slices of 1-2 mm thickness, and more than $90 \%$ of the cancerous content was a pre-requisite for microarray experiments. All examinations and management of the surgical specimens were carried out by 2 qualified pathologists (YYW and CYL). Written consent was obtained for all subjects before sample collection, and the study protocol was approved by Institute Review Board of Cathay General Hospital. The criteria of enrolment included incident/invasive breast cancers without neo-adjuvant therapy, no systemic spread (clinical stage I to III), no concurrent secondary malignancy, and less than 70 years of age.

Total RNA was extracted by TRIzol ${ }^{\circledR}$ reagent (Invitrogen, Carlsbad, CA) and the RNA was purified using RNeasy ${ }^{\circledR}$ mini kits (Qiagen, Germantown, MD). RNA integration was tested by gel electrophoresis. Affymetrix ${ }^{\circledR}$ (Affymetrix, Santa Clara, CA) GeneChip ${ }^{\circledR}$ Human Genome U133 plus 2.0 was used for the microarray experiment. Hybridization and scanning were performed according to a standard protocol. Images were scanned using GeneChip ${ }^{\circledR}$ Scanner 3000, and the scanned images were processed with GeneChip ${ }^{\circledR}$ Operating Software (GCOS). Robust multi-array average (RMA) algorithm was used to normalize 44 array chips [11]. For the 125 samples from China, raw expression files (CEL files) were downloaded from NCBI Gene Expression Omnibus (GSE5460) by using the same Affymetrix ${ }^{\circledR}$ U133 plus 2.0 arrays and normalized by RMA; details of the study are described elsewhere and the study was approved by local IRB [12]. The processed expression profiles of breast cancers from Taiwan and Mainland China were pooled together, and quantile normalization was performed to remove the batch effect between the breast cancer in Taiwanese patients and Chinese patients.

For relevant pathological features, estrogen receptor (ER) positivity was defined as the presence of at least $10 \%$ of nuclei with positive results of immunohistochemical (IHC) analysis, and breast samples displaying low ER positivity (1-9\% of nuclei with positive stains) were not assayed in current study. For HER2 status, the ASCO and CAP guidelines were followed; IHC $3+$ and IHC 2+ with fluorescence in-situ (FISH) hybridization amplification were considered to indicate HER2 over-expression. The modified Bloom-Richardson (Nottingham) system was used for grading breast cancers. The demographic features of the 169 Han Chinese patients with breast cancers are summarized in Table 1.

\section{Intrinsic gene lists and prototypical samples}

Three intrinsic signatures that defined 5 molecular subtypes (luminal A, luminal B, normal breast-like, HER2enriched, and basal-like) were Sørlie 500, Hu 306 and PAM50 $[3,4,6]$. These different sets of intrinsic genes presented a chronological evolution of molecular subtypes proposed by the Stanford group. The expression values of training samples deriving intrinsic signatures were downloaded from Stanford Genomics Breast Cancer Consortium and UNC Microarray Database (see Additional file 1 for details). Centroids were the mean expression values of intrinsic genes corresponding to each molecular subtype.

\section{Single sample prediction and systemic bias adjustment}

All intrinsic genes were mapped to the Affymetrix gene annotation file, and the data of the genes represented by 
Table 1 Demographic features of study population

\begin{tabular}{lccc}
\hline Source & $\begin{array}{c}\text { Taiwan } \\
\mathrm{n}=44\end{array}$ & $\begin{array}{c}\text { China } \\
\mathrm{n}=125\end{array}$ & $\begin{array}{c}\text { Total } \\
\mathrm{n}=169\end{array}$ \\
\hline ER & & & \\
$\quad$ Positive & $22(50 \%)$ & $74(59 \%)$ & $96(57 \%)$ \\
$\quad$ Negative & $22(50 \%)$ & $51(41 \%)$ & $73(43 \%)$ \\
HER2 & & & \\
$\quad$ Over-expressed & $21(48 \%)$ & $30(24 \%)$ & $51(30 \%)$ \\
$\quad$ Not & $23(52 \%)$ & $95(76 \%)$ & $118(70 \%)$ \\
Nuclear grade & & & \\
I & $3(7 \%)$ & $27(22 \%)$ & $30(18 \%)$ \\
II & $18(41 \%)$ & $31(25 \%)$ & $49(29 \%)$ \\
III & $23(52 \%)$ & $67(54 \%)$ & $90(53 \%)$ \\
Nodal status & & & \\
$\quad$ Positive & $23(52 \%)$ & $61(49 \%)$ & $84(50 \%)$ \\
$\quad$ Negative & $21(48 \%)$ & $64(51 \%)$ & $85(50 \%)$ \\
Lympovascular invasion* & & & \\
$\quad$ Positive & $27(63 \%)$ & $46(37 \%)$ & $73(43 \%)$ \\
$\quad$ Negative & $16(37 \%)$ & $79(63 \%)$ & $95(57 \%)$ \\
\hline *one missing value & & &
\end{tabular}

multiple-probesets were averaged (see Additional file 1 for details of mapping process). The 169 breast cancer specimens of the Han Chinese patients were assigned to 1 of the 5 molecular subtypes with the nearest centroid (single sample prediction). Spearman's rank correlation coefficients were used, and samples were designated as unclassified if correlation coefficients to all 5 centroids were less than 0.1 .

To enhance the comparability between the original studies deriving intrinsic genes and independent samples in current study, we applied 2 systemic bias-adjustment methods, mean-centring of genes and distance-weighted discrimination (DWD), to the expression data of Han Chinese breast cancers, as suggested by the investigators of the Stanford group $[13,14]$.

\section{Results}

\section{Distributions of molecular subtypes}

Table 2 shows the distribution of molecular subtypes under different combinations of intrinsic genes and adjustment methods. Without adjustment, both Sørlie 500 and $\mathrm{Hu} 306$ identified many unclassified samples, and the number of unclassified samples reduced rapidly when gene centring or DWD was applied, indicating the necessity of bias adjustment across microarray studies. The shift between the 2 luminal subtypes A and B was prominent with and without adjustment; however, the direction of this shift was unpredictable from the current study. On the other hand, PAM50 was less sensitive to systemic microarray bias correction. Regardless of the adjustment, the least changes were observed for the basal-like subtype across all 3 intrinsic genes. We also
Table 2 Molecular subtype distrubutions of 169 Han Chinese breast cancers with different intrinsic genes and adjustment

\begin{tabular}{|c|c|c|c|}
\hline Intrinsic genes & $\begin{array}{l}\text { Original data without } \\
\text { adjustment }\end{array}$ & $\begin{array}{l}\text { Mean-centring } \\
\text { of genes }\end{array}$ & $\begin{array}{c}\text { DWD } \\
\text { adjustment }\end{array}$ \\
\hline \multicolumn{4}{|l|}{ Sørlie 500} \\
\hline Luminal A & 36 & 69 & 70 \\
\hline Luminal B & 60 & 24 & 23 \\
\hline $\begin{array}{l}\text { Normal } \\
\text { breast-like }\end{array}$ & 4 & 11 & 15 \\
\hline Basal-like & 37 & 37 & 44 \\
\hline $\begin{array}{l}\text { HER2- } \\
\text { enriched }\end{array}$ & 2 & 21 & 13 \\
\hline Unclassified & 30 & 7 & 4 \\
\hline \multicolumn{4}{|l|}{ Hu 306} \\
\hline Luminal A & 84 & 58 & 57 \\
\hline Luminal B & 1 & 32 & 35 \\
\hline $\begin{array}{l}\text { Normal } \\
\text { breast-like }\end{array}$ & 0 & 8 & 10 \\
\hline Basal-like & 30 & 41 & 41 \\
\hline $\begin{array}{l}\text { HER2- } \\
\text { enriched }\end{array}$ & 16 & 29 & 25 \\
\hline Unclassified & 38 & 1 & 1 \\
\hline \multicolumn{4}{|l|}{ PAM50 } \\
\hline Luminal A & 70 & 56 & 51 \\
\hline Luminal B & 32 & 36 & 19 \\
\hline $\begin{array}{l}\text { Normal } \\
\text { breast-like }\end{array}$ & 10 & 6 & 27 \\
\hline Basal-like & 36 & 41 & 41 \\
\hline $\begin{array}{l}\text { HER2- } \\
\text { enriched }\end{array}$ & 19 & 30 & 31 \\
\hline Unclassified & 2 & 0 & 0 \\
\hline
\end{tabular}

noticed that PAM50 identified many normal breast-like tumours under DWD.

\section{Agreement between adjustment methods with the same intrinsic genes}

For Sørlie 500 and $\mathrm{Hu}$ 306, mean-centring of genes and DWD showed good agreement in subtype assignment (unweighted kappa: 0.83 and 0.95 , respectively). For PAM50, gene centring and original data showed the highest predictive consistency (unweighted kappa: 0.80), followed by gene centring and DWD, then original data and DWD (unweighted kappa: 0.67 and 0.66, respectively, Table 3).

\section{Agreement between intrinsic gene sets with the same adjustment}

Table 3 also shows pairwise agreement between the 3 intrinsic gene sets. When genes were mean-centred, Hu306 and PAM50 showed the highest agreement (unweighted kappa: 0.85), and the consistency dropped substantially (unweighted kappa: 0.67) when DWD was adopted for adjustment. Sørlie 500 intrinsic genes 
Table 3 Agreement between intrinsic gene sets and adjustment methods

\begin{tabular}{|c|c|c|c|c|c|}
\hline Intrinsic genes & Kappa* & $95 \% \mathrm{Cl}$ & Adjustment method & Kappa* & $95 \% \mathrm{Cl}$ \\
\hline Sørlie 500 & & & Gene centring & & \\
\hline Original data vs. gene centring & 0.51 & $0.43-0.60$ & Sørlie 500 vs. Hu 306 & 0.58 & $0.50-0.67$ \\
\hline Original data vs. DWD & 0.49 & $0.41-0.57$ & Sørlie 500 vs. PAM50 & 0.52 & $0.43-0.61$ \\
\hline Gene centring vs. DWD & 0.83 & $0.77-0.90$ & Hu306 vs. PAM50 & 0.85 & $0.79-0.91$ \\
\hline Hu 306 & & & DWD adjusted & & \\
\hline Original data vs. gene centring & 0.51 & $0.43-0.58$ & Sørlie 500 vs. Hu 306 & 0.56 & $0.47-0.65$ \\
\hline Original data vs. DWD & 0.5 & $0.42-0.58$ & Sørlie 500 vs. PAM50 & 0.55 & $0.47-0.64$ \\
\hline Gene centring vs. DWD & 0.95 & $0.92-0.99$ & Hu306 vs. PAM50 & 0.67 & $0.59-0.76$ \\
\hline \multicolumn{6}{|l|}{ PAM50 } \\
\hline Original data vs. gene centring & 0.8 & $0.73-0.87$ & & & \\
\hline Original data vs. DWD & 0.66 & $0.58-0.75$ & & & \\
\hline Gene centring vs. DWD & 0.67 & $0.58-0.75$ & & & \\
\hline
\end{tabular}

showed only substantial agreement with $\mathrm{Hu} 306$ and PAM50 under gene centring or DWD (see Additional file 2 for supplementary Table S2).

\section{Clinical features of molecular subtypes}

The breast cancer samples assigned to identical subtypes with both $\mathrm{Hu} 306$ and PAM50 intrinsic genes were retrieved. These included samples from 150 (89\%) and 126 (75\%) of the 169 study subjects under gene centring and DWD adjustment, respectively. In the most stringent conditions, 117 (69\%) samples were consistently assigned to identical subtypes under both DWD and gene centring. Their clinical features are presented in Table 4 (supplementary Table S1 for molecular subtypes stratified by clinical phenotypes); luminal B tended to show a higher nucler grade and HER2 over-expression than luminal A did. No basal-like breast tumours were ER positive, and most HER2-enriched breast tumours showed HER2 over-expression and were clinically ER negative, whereas, only two-thirds of tumours that showed ER negativity/HER2 over-expression were predicted as HER2-enriched molecular subtype.

For 44 Taiwanese breast cancers, clinical follow-up was available up to 62 months (median:44.5). There were 10 events of distant metastasis or mortality attributed to breast cancers. Figure 1(a) showed that there was no significant event-free survival difference among the 5 molecular subtypes (log-rank test: 0.13, with $\mathrm{Hu} 306$ intrinsic genes and gene-centring adjustment), better prognosis of luminal A and compromised survival of

Table 4 Clinical features of agreeing samples between Hu 306 and PAM50 ( $\mathrm{n=117}$ with both mean-centring and DWD adjustment)

\begin{tabular}{|c|c|c|c|c|c|}
\hline \multirow[t]{2}{*}{ Clinical factor } & \multicolumn{5}{|c|}{ Molecular subtype } \\
\hline & Luminal A & Luminal B & Normal-breast like & Basal-like & HER2-enriched \\
\hline \multicolumn{6}{|l|}{ ER } \\
\hline Positive & 35 & 15 & 2 & 0 & 3 \\
\hline Negative & 0 & 1 & 4 & 39 & 18 \\
\hline \multicolumn{6}{|l|}{ HER2 } \\
\hline over-expressed & 2 & 5 & 2 & 2 & 18 \\
\hline not over-expressed & 33 & 11 & 4 & 37 & 3 \\
\hline \multicolumn{6}{|l|}{ Nuclear grade } \\
\hline 1 & 17 & 0 & 1 & 0 & 0 \\
\hline$\|$ & 16 & 4 & 2 & 2 & 4 \\
\hline III & 2 & 12 & 3 & 37 & 17 \\
\hline \multicolumn{6}{|l|}{ Nodal status } \\
\hline Positive & 16 & 9 & 5 & 10 & 14 \\
\hline Negative & 19 & 7 & 1 & 29 & 7 \\
\hline \multicolumn{6}{|c|}{ Lymphovascular invasion* } \\
\hline Positive & 14 & 11 & 4 & 11 & 13 \\
\hline Negative & 21 & 5 & 2 & 28 & 8 \\
\hline
\end{tabular}

*One missing value 


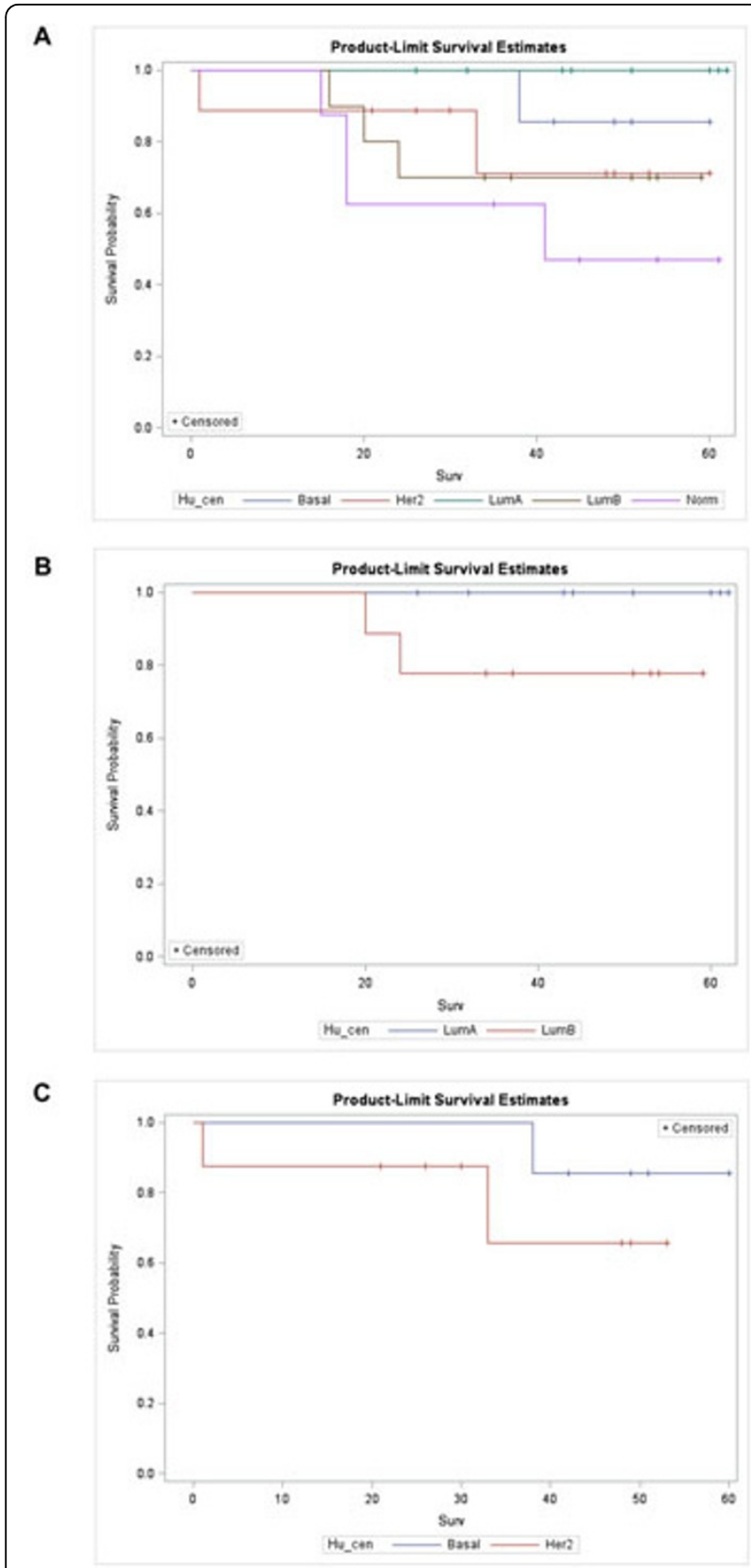

Figure 1 Survival analysis of 44 Taiwanese breast cancers with $\mathrm{Hu}$ 306 intrinsic genes and mean-centring adjustment (a). Subgroup analysis in ER-positive breast cancers ( $n=19$ ), only luminal A and B subtypes displayed due to the sparseness of other molecular subtypes (b). Subgroup analysis in ER-negative breast cancers ( $n=15)$, only basal-like and HER2-enriched subtypes displayed due to the sparseness of other molecular subtypes (c). (abbreviations: LumA: luminal A, LumB: luminal B, Her2, HER2-enriched, basal: basallike, Norm: normal breast-like subtype, Hu_cen: Hu 306 intrinsic genes with mean-centring adjustment).

luminal B and HER2-enriched subtype was apparent though. For ER-positive patients, luminal A outperformed luminal B while in ER-negative cancers, the prognosis of basal-like subtype was better than HER2enriched subtype for Taiwanese breast cancers (Figure 1 (b) and 1 (c), respectively).

\section{Discussion}

In the current study, we evaluated the trans-ethnic applicability of breast cancer molecular subtypes for independent samples obtained from people of the Han Chinese origin. Three distinct intrinsic gene sets were used, and the systemic microarray bias was accounted for by carrying out mean-centring of genes or DWD. We found that without adjustment, Sørlie 500 and $\mathrm{Hu}$ 306 signatures had a higher proportion of unclassified cases, thereby highlighting the importance of eliminating systemic bias and enhancing comparability across microarray studies. This was further supported by the high kappa statistics between DWD and gene centring when Sørlie 500 and $\mathrm{Hu} 306$ intrinsic genes were used. Among all the 3 intrinsic gene sets, PAM50 was less sensitive to systemic adjustment but showed more normal breast-like samples (especially with DWD).

The number of breast cancer samples predicted as normal breast-like subtype was largely influenced by intrinsic genes used and adjustment method. Notably, normal breast-like centroid in PAM50 was derived from 29 normal breast samples and should be treated as an internal quality control rather than a breast cancer intrinsic variation in $\mathrm{Hu} 306$ and Sørlie 500 [6]. In this sense, none of our samples should be predicted as normal breast-like subtype with PAM50. In our study, the higher number of samples catogorised as the normal breast-like subtype by PAM50 indicates possible normal breast tissue contamination without laser capture microdissection, or dubious predictive accuracy of PAM50 normal breast-like centroid derived from unrepresentative or limited number of normal breast samples. Another possible explanation is that gene expression values were obtained by microarray experiments rather than reserve transcriptase polymerase chain reaction (RT-PCR) used by the PAM50 signature, and this led to some discrepancies in measurements.

We next compared the pairwise consistency between the distinct intrinsic gene sets, under the same adjustment manner. With gene centring, $\mathrm{Hu} 306$ and PAM50 showed the highest consistency (unweighted kappa: 0.85 ), or nearly $90 \%$ of the assayed samples were identified to be of the same subtype. Hu 306 and PAM50 still showed the highest agreement when adjusted by DWD, but only substantial agreement (unweighted kappa: 0.67) was observed. Because Sørlie 500 appeared the first in all 3 intrinsic signatures, many of its clone ID identifiers had difficulties mapping to the latest HUGO gene symbol and modern microarray platform used in current study. Investigators of the Stanford group also suggested the use of the most 
recently updated PAM50 during molecular subtyping of clinical samples $[6,13]$. Here, we discourage the use of Sørlie 500 gene set for intrinsic subtyping. Regarding adjustment method, gene-centring rather than DWD might deliver more optimisitc results since fewer normal breast-like samples were predicted, especially when PAM50 intrinsic genes were used.

Some researchers might tend to use clinical phenotypes as surrogates for breast cancer molecular subtypes such that ER-positive tumours were analogous to luminal subtype, tumours with ER-negativity/HER2 over-expression were analogous to HER2-enriched subtype, and tumours with ER-negativity/HER2 negativity were analogous to basal-like breast cancer $[15,16]$. Supplementary Table S3 (Additional file 3) showed the intrinsic subtype distrubutions stratified by so-called "IHC subtype" in our study and as expected, there was a substantial disagreement between molecular subtype defined by intrinsic genes and subtype determined by conventional IHC methods alone. However, molecular subtypes were determined to be independent of pathological markers and the investigtors of PAM50 also argued that ER and HER2 status alone were not accurate surrogates for 'true' intrinsic subtype status; for instance, only $64 \%$ of cases with HER2 over-expression were designated as HER2-enriched subtype in their study [6]. The discrepancy between clinical HER2 phenotype and molecular HER2-enriched subtype raised the serious concern that whether these patients should be managed according to IHC/FISH results or gene expression profiles and this dispute remained inconclusive. In table 4 we summarized pathological features of each molecular subtype from samples presistantly predicted into identical subtype by Hu 306 and PAM50 under both DWD and gene-centring adjustment (roughly $70 \%$ of all assayed samples). As expected, luminal B showed more aggressive behaviors by traditional prognostic features, all basal-like tumros were ER negative, most HER2-enriched tumors showed IHC/ FISH HER2 overe-expression, whereas only two-thirds of tumours with clinical ER-negativity/HER2 over-expression were predicted as HER2-enriched subtype according to the gene expression assays, and these findings were grossly in concordance with our knowledge about each breast cancer molecular subtype. For 44 Taiwanese breast cancers with survival data, we did find a trend toward good prognosis for luminal A subtype and worse prognosis for luminal B and HER2-enriched subtype, and a survival benefit of basal-like over HER2-enriched subtype was also observed, especially when subgroup analysis was performed according by IHC ER status.

Our study had some limitations. First, we did not have a sufficient sample size to determine the prognostic value of each molecular sutbype upon breast cancer survival and multi-variate analysis incorporating clinical and pathological factors was not possible. Second, a prospective study design is needed to eliminate selection bias and may be help to incorporate breast cancer molecular subtypes into clinical decision makings.

\section{Conclusions}

In the current study we evaluted the prediction consistency and clinical presentations of breast cancer molecular signatures trans-ethnically for Han Chinese population. We found that with proper adjustments to enhance comparability across microarray studies, the predictive consistency between PAM50 and Hu 306 was achieved for nearly $90 \%$ of our independent breast cancer samples, and disparities in the associated clinical and pathological features were observed between distinct molecular subtypes. A trend of prognostic disparity was also observed from intrinsic subtypes among Taiwanese breast cancers, and provides an opportunity for developing risk prediction models and dissecting the heterogeneity within ER positive and negative breast cancers respectively. Further work to evaluate the relevance of molecular subtypes and survival should be initiated using more clinical samples with longer follow-up of patients.

\section{Additional material}

Additional file 1: Supplementary materials and methods This file
included the mapping process of intrinsic genes to Affymetrix ${ }^{\circledR} \oplus$
probesets, and the source of Chinese breast cancer microarrays. Table S1
summarized intrinsic subtype distributions stratified by clinical
phenotypes.
Additional file 2: Supplementary Table S2 Supplementary Table S2
contained pairwise comparisons between 3 intrinsic gene lists for the
assignment of the samples.
Additional file 3: Supplementary Table S3 Table S3 showed the
distributions of molecular subtypes defined by intrinsic genes (Hu 306
and PAM50 with gene-centring and DWD) stratified by subtypes defined
by IHC results.

\section{Abbreviations}

ER: estrogen receptor; HER2: human epidermal growth factor receptor 2; DWD: distance-weighted discrimination; SSP: single sample predictor; IHC: immunohistochemical; RT-PCR: reserve transcriptase polymerase chain reaction

\section{Acknowledgements}

The work was supported in part by Cathay Medical Research Institute grant CGH-MR-9609, National Taiwan University grant 95R0066-BM01-01 and 98HM0001 and National Science Council grant 100-2314-B-281-005-MY2. This article has been published as part of Journal of Translational Medicine Volume 10 Supplement 1, 2012: Selected articles from the Organisation for Oncology and Translational Research (OOTR) 7th Annual Conference. The full contents of the supplement are available online at http://www.translationalmedicine.com/supplements/10/S1.

\section{Author details}

${ }^{1}$ Graduate Institute of Biomedical Electronics and Bioinformatics, National Taiwan University, Taipei City, Taiwan. ${ }^{2}$ Department of Surgery, Cathay General Hospital SiJhih, New Taipei City, Taiwan. ${ }^{3}$ School of Medicine, Fu-Jen 
Catholic University, New Taipei City, Taiwan. ${ }^{4}$ School of Medicine, Taipei Medical University, Taipei City, Taiwan. ${ }^{5}$ Department of Surgery, Cathay General Hospital, Taipei City, Taiwan. ${ }^{6}$ Department of Pathology, Cathay General Hospital SiJhih, New Taipei City, Taiwan. ${ }^{7}$ Graduate Institute of Physiology, National Taiwan University, Taipei City, Taiwan.

\section{Authors' contributions}

$\mathrm{CCH}$ initiated the study and drafted the manuscript. SHT, HHL, JYJ and CSH participated in the study design, sample collection and shared experts' opinions. YYW and CYL handled the frozen samples and performed all pathological examinations. JSL provided statistical help and interpretation of results. LCL carried out microarray experiments. EYC conceived the whole study and took the responsibility of corresponding author. All authors read and approve the final manuscript.

\section{Competing interests}

The authors declare that they have no competing interests.

Published: 19 September 2012

\section{References}

1. Perou CM, Sørlie T, Eisen MB, van de Rijn M, Jeffrey SS, Rees CA, Pollack JR, Ross DT, Johnsen H, Akslen LA, Fluge O, Pergamenschikov A, Williams C, Zhu SX, Lønning PE, Børresen-Dale AL, Brown PO, Botstein D: Molecular portraits of human breast tumours. Nature 2000, 406:747-452.

2. Sørlie T, Perou CM, Tibshirani R, Aas T, Geisler S, Johnsen H, Hastie T, Eisen MB, van de Rijn M, Jeffrey SS, Thorsen T, Quist $H$, Matese JC, Brown PO, Botstein D, Lønning PE, Børresen-Dale AL: Gene expression patterns of breast carcinomas distinguish tumor subclasses with clinical implications. Proc Natl Acad Sci U S A 2001, 98:10869-10874.

3. Sorlie T, Tibshirani R, Parker J, Hastie T, Marron JS, Nobel A, Deng S, Johnsen H, Pesich R, Geisler S, Demeter J, Perou CM, Lønning PE, Brown PO, Børresen-Dale AL, Botstein D: Repeated observation of breast tumor subtypes in independent gene expression data sets. Proc Natl Acad Sci U S A 2003, 100:8418-8423.

4. Hu Z, Fan C, Oh DS, Marron JS, He X, Qaqish BF, Livasy C, Carey LA, Reynolds E, Dressler L, Nobel A, Parker J, Ewend MG, Sawyer LR, Wu J, Liu Y, Nanda R, Tretiakova M, Ruiz Orrico A, Dreher D, Palazzo JP, Perreard L, Nelson E, Mone M, Hansen H, Mullins M, Quackenbush JF, Ellis MJ, Olopade OI, Bernard PS, Perou CM: The molecular portraits of breast tumors are conserved across microarray platforms. BMC Genomics 2006, 7:96.

5. Sotiriou C, Neo SY, MCShane LM, Korn EL, Long PM, Jazaeri A, Martiat $P$, Fox SB, Harris AL, Liu ET: Breast cancer classification and prognosis based on gene expression profiles from a population-based study. Proc Natl Acad Sci U S A 2003, 100:10393-10398.

6. Parker JS, Mullins M, Cheang MC, Leung S, Voduc D, Vickery T, Davies $S$, Fauron C, He X, Hu Z, Quackenbush JF, Stijleman IJ, Palazzo J, Marron JS, Nobel AB, Mardis E, Nielsen TO, Ellis MJ, Perou CM, Bernard PS: Supervised risk predictor of breast cancer based on intrinsic subtypes. J Clin Oncol 2009, 27:1160-1167.

7. Lusa L, McShane LM, Reid JF, De Cecco L, Ambrogi F, Biganzoli E, Gariboldi M, Pierotti MA: Challenges in projecting clustering results across gene expression-profiling datasets. J Natl Cancer Inst 2007, 99:1715-1723.

8. Weigelt B, Mackay A, A'hern R, Natrajan R, Tan DS, Dowsett M, Ashworth A, Reis-Filho JS: Breast cancer molecular profiling with single sample predictors: a retrospective analysis. Lancet Oncol 2010, 11:339-349.

9. Fan C, Oh DS, Wessels L, Weigelt B, Nuyten DS, Nobel AB, van't Veer $L$, Perou CM: Concordance among gene-expression-based predictors for breast cancer. N Engl J Med 2006, 355:560-569.

10. Mackay A, Weigelt B, Grigoriadis A, Kreike B, Natrajan R, A'Hern R, Tan DS, Dowsett M, Ashworth A, Reis-Filho JS: Microarray-based class discovery for molecular classification of breast cancer: analysis of interobserver agreement. J Natl Cancer Inst 2011, 103:662-673.

11. Irizarry RA, Hobbs B, Collin F, Beazer-Barclay YD, Antonellis KJ, Scherf U, Speed TP: Exploration, normalization, and summaries of high density oligonucleotide array probe level data. Biostatistics 2003, 4:249-264.

12. Lu X, Lu X, Wang ZC, Iglehart JD, Zhang X, Richardson AL: Predicting features of breast cancer with gene expression patterns. Breast Cancer Res Treat 2008, 108:191-201.
13. Sørlie T, Borgan E, Myhre S, Vollan HK, Russnes H, Zhao X, Nilsen G, Lingjaerde OC, Børresen-Dale AL, Rødland E: The importance of genecentring microarray data. Lancet Oncol 2010, 11:719-720.

14. Benito M, Parker J, Du Q, Wu J, Xiang D, Perou CM, Marron JS: Adjustment of systematic microarray data biases. Bioinformatics 2004, 20:105-114.

15. Pusztai L, Mazouni C, Anderson K, Wu Y, Symmans WF: Molecular classifications of breast cancer: limitations and potential. Oncologist 2006, 11:868-877.

16. Weigelt B, Reis-Filho JS: Molecular profiling currently offers no more than tumour morphology and basic immunohistochemistry. Breast Cancer Res 2010, 12(Suppl 4):S5.

doi:10.1186/1479-5876-10-S1-S10

Cite this article as: Huang et al:: Prediction consistency and clinical presentations of breast cancer molecular subtypes for Han Chinese population. Journal of Translational Medicine 2012 10(Suppl 1):S10.

\section{Submit your next manuscript to BioMed Central and take full advantage of:}

- Convenient online submission

- Thorough peer review

- No space constraints or color figure charges

- Immediate publication on acceptance

- Inclusion in PubMed, CAS, Scopus and Google Scholar

- Research which is freely available for redistribution

Submit your manuscript at www.biomedcentral.com/submit
Ciomed Central 